\title{
Mechanisms of pattern formation in grazing-incidence ion bombardment of $\operatorname{Pt}(111)$
}

\author{
Henri Hansen, ${ }^{1}$ Alex Redinger, ${ }^{1}$ Sebastian Messlinger, ${ }^{1, *}$ Georgiana Stoian, ${ }^{1,}$ Yudi Rosandi, ${ }^{2}$ Herbert M. Urbassek, ${ }^{2}$ \\ Udo Linke, ${ }^{3}$ and Thomas Michely ${ }^{1}$ \\ ${ }^{1}$ I. Physikalisches Institut, RWTH Aachen University, 52056 Aachen, Germany \\ ${ }^{2}$ Fachbereich Physik, Universität Kaiserslautern, Erwin-Schrödinger-Straße, D-67663 Kaiserslautern, Germany \\ ${ }^{3}$ Institute of Thin Films and Interfaces, Forschungszentrum Jülich GmbH, 52425 Jülich, Germany
}

(Received 2 January 2006; revised manuscript received 2 May 2006; published 16 June 2006)

\begin{abstract}
Ripple patterns forming on $\mathrm{Pt}(111)$ due to $5 \mathrm{keV} \mathrm{Ar}^{+}$grazing-incidence ion bombardment were investigated by scanning tunneling microscopy in a broad temperature range from 100 to $720 \mathrm{~K}$ and for ion fluences up to $3 \times 10^{20}$ ions $/ \mathrm{m}^{2}$. A detailed morphological analysis together with molecular dynamics simulations of single ion impacts allow us to develop atomic scale models for the formation of these patterns. The large difference in step edge versus terrace damage is shown to be crucial for ripple formation under grazing incidence. The importance of distinct diffusion processes-step adatom generation at kinks and adatom lattice gas formation-for temperature dependent transitions in the surface morphology is highlighted. Surprisingly, ion bombardment effects like thermal spike induced adatom production and planar subsurface channeling are important for pattern ordering.
\end{abstract}

DOI: 10.1103/PhysRevB.73.235414

PACS number(s): 81.16.Rf, 79.20.Rf, 79.20.Ap, 61.80.Jh

\section{INTRODUCTION}

Pattern formation on crystalline and amorphous solids using ion bombardment has become a topic of rapidly increasing interest. One reason is certainly the potential of such nanoscale patterns (linear dimensions of the order of $10-100 \mathrm{~nm}$ ) for applications. With the use of suitable ion sources, they can be produced relatively cheaply and uniformly over large surface areas (linear dimensions of the order of $0.1 \mathrm{~m}$ ). Currently their use for manipulating magnetism, ${ }^{1,2}$ for the ordered adsorption of large molecules, ${ }^{3}$ for tuning the chemical reactivity of catalytically active surfaces, ${ }^{4}$ for optoelectronic devices, ${ }^{5}$ for manipulating film texture ${ }^{6}$ or for use as molding templates ${ }^{7}$ are explored. Deciphering the formation mechanisms of these patterns at the atomic scale is a fascinating and demanding problem. Once solved, it would allow us to tune their properties (lateral dimension, uniformity, shape, and aspect ratio) in a more precise and efficient way than nowadays, with ensuing consequences for their applicability. It is our goal in this work to contribute to the atomic scale understanding of the surface pattern formation mechanisms by ion bombardment.

Two groups of patterns result from ion bombardment. Dot or pit arrangements at normal or slightly off-normal beam incidence and ripple patterns at a significant off normal (ripple wave vector parallel to the projection of the ion beam on the surface) or grazing beam incidence (ripple wave vector normal to the projection of the ion beam). We note that ripple patterns may also be formed by normal incidence ion bombardment of low symmetry, anisotropic crystalline substrates. ${ }^{8}$ There is a growing consensus that the formation of dot and pit patterns of crystalline materials (remaining also crystalline during bombardment) - in particular, metals - can be explained in terms of elementary atomic processes. ${ }^{9-19}$ At sufficiently high temperatures, the formation of pit patterns can be attributed to the creation of mobile surface vacancies and vacancy clusters, their aggregation to islands, and the action of a step edge barrier for surface vacancies. ${ }^{10,12-20}$ Coarsening behavior could be linked to material transport along step edges and the high temperature breakdown of pattern formation to the onset of step atom detachment. ${ }^{13}$ Toward lower temperatures, a transition from pit to dot patterns is observed, which is linked to the mobility of adatoms and adatom clusters generated during bombardment, whereas surface and subsurface vacancy and vacancy cluster mobility ceases. ${ }^{10,12,20}$ Dot patterns on amorphous or ion beam amorphized surfaces (e.g., semiconductor surfaces) are usually described in terms of continuum theory. ${ }^{5,21}$ This holds as well for the dot pattern created at off-normal incidence, but with sample rotation. ${ }^{22,23}$ The evolution of these highly ordered patterns and their symmetry is not fully understood, but significant advances in their theoretical description were achieved. ${ }^{24}$

The situation is somewhat different and less conclusive for off-normal and grazing-incidence ion bombardment. The morphological evolution of (0001)-graphite ${ }^{25}$ (amorphizing during bombardment) and $\mathrm{Cu}(001)^{26}$ for not too large ion fluences (i.e., ion beam exposure times) is apparently adequately described by linear continuum theory. However, there are examples that exhibit an increase of the ripple wavelength with ion fluence according to a power law already in the early stages of morphological evolution, e.g., for $\mathrm{Ag}(001), \mathrm{Cu}(110)$, and polycrystalline metal films. ${ }^{8,27,28} \mathrm{The}$ increase of the ripple wavelength with fluence is not described in linear continuum theory. ${ }^{29}$ Attempts were made to incorporate into the continuum theory description ${ }^{27,30,31}$ effects of the crystal structure-e.g., the step edge barrier for adatoms or diffusion anisotropy-and nonlinear terms to account for kinetic roughening. Whether the additional terms in the continuum theory description are sufficient for an adequate description of ripple patterns on anisotropic substrates or of ripple patterns that exhibit coarsening right from the beginning of their morphological evolution is not yet settled.

In contrast to normal incidence ion bombardment, much less work describes the morphological evolution of ripple patterns in terms of atomic scale processes and links specific features to specific processes. In particular, the onset of 
ripple pattern formation is not yet well understood. Continuum theory postulates as the key destabilizing mechanism in ripple pattern formation the curvature dependent sputtering yield. 29,30,32 Physically, it is based on Sigmund's finding ${ }^{33}$ that the impinging ions deliver more energy to the surface in depressions compared to elevations. This implies a smaller sputtering yield of elevations, which in turn gives rise to surface destabilization. The Sigmund mechanism explicitly neglects the crystalline structure. It can only be considered to be appropriate for the radii of curvature that are large compared to the diameter of the impact influenced zone. Beyond this continuum theory postulate there is, to the authors' best knowledge, only the work of van Dijken et al. ${ }^{34}$ which gives an atomic scale explanation of the formation of shallow, only a few layers deep ripples ("nanogrooves") upon grazing incidence ion bombardment. Several aspects of the nanogroove formation scenario will be discussed in relation to the present work in Sec. VI.

Here we investigate the atomic scale formation mechanisms of ripple formation on $\mathrm{Pt}(111)$ under $5 \mathrm{keV} \mathrm{Ar}^{+}$ion bombardment at a grazing incidence $\left(\vartheta=83^{\circ}\right)$ along the

[1112] direction. Under these conditions, it is found that first a pattern of elongated vacancy islands (long, monolayer deep depressions, also named vacancy grooves) is formed. With increasing ion exposure, this pattern transforms into a multilayer pattern of ripples. The structure formation sets in at low ion fluences and-contrary to the aforementioned nanogroove case-the amplitude of the pattern rapidly increases with ion fluence according to a power law. ${ }^{35}$ Surprising and unique elements are found to be relevant for ripple formation, among them (i) the irrelevance of vacancy island nucleation; (ii) an extreme difference in sputtering yields for terrace impacts and step edge impacts; (iii) an athermal adatom mechanism for the alignment of elongated vacancy islands; and (iv) the importance of planar subsurface channeling at elevated temperatures, which we addressed already in a recent letter. ${ }^{36} \mathrm{~A}$ coherent picture of ripple formation at grazing incidence is given later, which emphasizes the role of distinct atomic scale diffusion processes in their formation.

The outline of the manuscript is as follows. After a brief presentation of the experimental and simulation procedures (in Sec. II) relevant information on diffusion processes and ion surface interaction for the specific system under concern-Pt(111)—is briefly summarized in Sec. III. Section IV gives an overview on the temperature dependence of ripple formation on $\mathrm{Pt}(111)$, while the distinct temperature regimes of pattern formation are discussed in detail in Secs. $\mathrm{V}$ and VI. Finally, the paper ends with Sec. VII containing a brief discussion of the roughness evolution of the patterns.

\section{EXPERIMENTAL AND SIMULATION PROCEDURES}

The experiments were performed in an ultrahigh vacuum (UHV) variable temperature STM apparatus with a base pressure in the $10^{-11}$ mbar range. ${ }^{37}$ Sample cleaning was accomplished by cycles of ion bombardment at room temperature (r.t.) and flash annealing to $1273 \mathrm{~K}$. For the grazing incidence ion experiments, the clean surface was exposed to a mass separated $5 \mathrm{keV} \mathrm{Ar}^{+}$ion beam incident along the [1112] direction at an angle of $\vartheta=83^{\circ}$ with respect to the surface normal at various temperatures and for various exposure times. The ion flux was calibrated prior to each experiment with a Faraday cup moved to the sample position and controlled after the end of the bombardment time. The ion flux used was $1.8 \times 10^{16}$ ions $\mathrm{m}^{-2} \mathrm{~s}^{-1}$ (corresponding to 1.2 $\times 10^{-3} \mathrm{MLE} / \mathrm{s}$ ), if not otherwise specified. For simplicity in the following, the ion fluence $F$ (the product of ion flux and exposure time) is specified in monolayer equivalents (MLE), where $1 \mathrm{MLE}=1.504 \times 10^{19} \mathrm{ions} / \mathrm{m}^{2}$. This quantity is numerically identical to the areal density of $\mathrm{Pt}$ atoms on the (111) surface usually specified as one monolayer (ML). At the end of the bombardment time interval the sample was quenched to r.t. This freezing temperature is sufficient, as with the end bombardment all mobile species (adatoms, vacancies, small clusters) are rapidly incorporated into steps, which are immobile on the time scale of hours (no fluctuations) below $400 \mathrm{~K}$. For experiments performed below r.t., the sample is quenched either to about $100 \mathrm{~K}$ for a bombardment temperature above $150 \mathrm{~K}$ or to about $40 \mathrm{~K}$ for even lower bombardment temperatures. STM imaging started after temperature stabilization, typically within $15 \mathrm{~min}$ after the end of the ion exposure. STM images in this paper are displayed as grayscale topographs, if not specified otherwise.

The morphology in grazing incidence ion bombardment depends critically on the angle $\vartheta$. Therefore it is crucial to control and reproduce this angle precisely. To this purpose the sample holder plate can be tilted in an axis normal to the ion beam through a gear mechanism changing $\vartheta$ by $0.8^{\circ}$ per turn. The absolute value of $\vartheta$ is measured through the position of the reflected ion beam and its shift with $\vartheta$ with the help of a second Faraday cup located $47 \mathrm{~mm}$ behind the sample in the beam direction, which can be moved up and down normal to the ion beam in the plane formed by the beam and the surface normal. The beam divergence is around $1^{\circ}$, the precision of the absolute value of $\vartheta$ is better than $1^{\circ}$ and the reproducibility of $\vartheta$ is $\approx 0.2^{\circ}$.

The wavelength analysis of the ripple patterns formed due to ion bombardment was performed with two different methods yielding similar results within an accuracy of better than $10 \%$. First, the height-height correlation function $G(x, y)$ $=\left\langle h^{\prime}(\widetilde{x}, \widetilde{y}) h^{\prime}(\widetilde{x}+x, \widetilde{y}+y)\right\rangle_{\widetilde{x}, \tilde{y}}$ with $h^{\prime}(\widetilde{x}, \widetilde{y})=h(\widetilde{x}, \widetilde{y})-\bar{h}$ was determined for representative STM topographs. Here $\bar{h}$ is the average height. As a measure of the wavelength $\lambda$ we used the separation between the two first minima in the direction normal to the ripple pattern. Second, histograms of the separation of minima in line profiles taken normal to the ripple pattern in the STM topographs were established with sufficient statistics. The average separation was taken as the wavelength $\lambda$ of the ripple pattern. The standard deviations of the separation histograms are a measure of pattern regularity.

In order to obtain an atomistic insight into the processes occurring under ion impact, a series of molecular-dynamics simulations were performed. ${ }^{38,39}$ We use a Pt (111) crystallite, which consists of 14240 atoms, arranged in 11 layers, each containing 1344 atoms. Its (111) surface is free and 


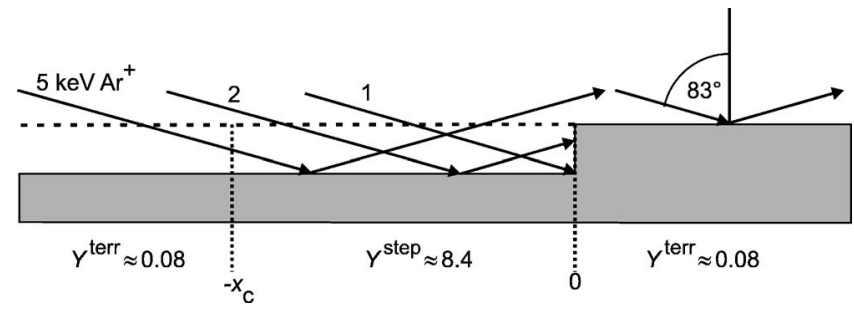

FIG. 1. Schematic side view of experimental geometry during ion exposure. Ions crossing the dashed line in front of an illuminated step at a distance $\xi$ within the interval $\left[-x_{c}, 0\right]$ give rise to the large step edge sputtering yield $Y^{\text {step }} \approx 8.4$, whereas all other ions cause only negligible sputtering with $Y^{\text {terr }} \approx 0.08$.

relaxed; the other five sides contain three layers of damped atoms in order to simulate the environment of the crystallite; the outermost layers are fixed. ${ }^{40}$ We employ a many-body interaction potential ${ }^{41}$ to describe the Pt interatomic interaction, which has been splined at high energies to the $\mathrm{ZBL}^{42}$ potential. The Ar-Pt interaction is modeled to be purely repulsive according to the $\mathrm{ZBL}$ potential. We simulated the processes occurring after ion impact for at least $20 \mathrm{ps}$. The simulation data shown here refer to a target temperature of $0 \mathrm{~K}$, unless otherwise indicated. A number of simulations have also been performed at $550 \mathrm{~K}$ in order to compare to experiments performed at $550 \mathrm{~K}$. We use velocity scaling to reach this temperature in the target and let it relax for at least $25 \mathrm{ps}$ to reach equilibrium. We note that for these simulations it was essential to fix the bottommost layer of the crystallite in order to stabilize it against "floppy" longwavelength vibrational and torsional modes.

\section{ATOMIC SCALE DAMAGE OF GRAZING INCIDENCE IONS AND DIFFUSION PROCESSES ON PT(111)}

In the present section we summarize the knowledge on the interaction of ions with $\operatorname{Pt}(111)$ and diffusion processes on this surface, which is a prerequisite for an adequate discussion of ripple formation in the subsequent sections.

Figure 1 exhibits a schematic side view of the bombardment geometry. By molecular-dynamics (MD) simulations and STM experiments, ${ }^{39}$ it was found that ascending step edges illuminated by the ion beam possess a dramatically enhanced sputtering yield compared to terraces. To be more precise, ions crossing the dashed line in Fig. 1 in front of the ascending step at a distance $\xi \in\left[-x_{c}, 0\right]$ possess an average sputtering yield of about 8.4 for the ion and surface parameters used in the present experiment. In this geometric model these ions hit the step edge either directly (trajectory 1 in Fig. 1) or after reflection on the lower terrace (trajectory 2 in Fig. 1). For convenience, lengths on the surface will be denoted in dimensionless units, $\xi=x / \Delta x$, where $\Delta x=2.40 \AA$ is

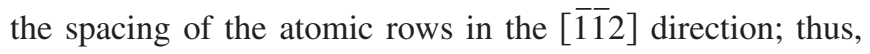
on $\operatorname{Pt}(111)$ for $\vartheta=83^{\circ}, x_{c}$ corresponds to a value of $x_{c}$ $=x / \Delta x=15.4$ atomic rows. The sputtering yield for these ions is consequently denoted as the step edge sputtering yield $Y^{\text {step }}$. The MD simulations also indicate that ions hitting the step edge right at the bottom (as trajectory 1) possess a sig- nificant probability for subsurface planar channeling between the topmost and the underneath atomic layer, i.e., for guided motion of the ions between these layers. ${ }^{36}$ In contrast, for ions hitting the surface on the terrace, a low terrace sputtering yield of $Y^{\text {terr }} \approx 0.08$ was found experimentally. Thus $Y^{\text {step }}$ exceeds $Y^{\text {terr }}$ by a factor of about 100 . It should be noted that this yield enhancement at step edges is independent of the ion flux enhancement onto step edges. Indeed, due to the width $x_{c}$ of the stripe in front of an ascending step edge, on top of the yield difference a step edge atom receives a flux 15 times larger than a terrace atom under the conditions used here.

Molecular dynamics simulations yield additional insight into the damage patterns evolving from terrace and step edge impacts. In consequence of a typical step edge impact after the cascade phase, a well developed thermal spike evolves. Due to viscous flow of the melt onto the surface, a rim of adatom clusters develops, which contains in the average 23 atoms. In this rim the adatoms are distributed in a broad cluster size range from single adatoms to clusters containing more than 50 atoms. The rim surrounds the central vacancy cluster core. It contains, on average, about 31 surface vacancies from the topmost surface level (frequently connected to the lower terrace) and lower levels. The majority of the surface vacancies are usually found in one or two large core clusters, but also small vacancy clusters and single surface vacancies are observed. The difference between the numbers of adatoms and vacancies created is roughly the amount of sputtered atoms, which was measured to be 8.3 atoms in our simulations. Due to the localization of the energy deposition very close to the surface, generally the amount of bulk damage (bulk vacancies, bulk vacancy clusters, and interstitial atoms) is small compared to the visible surface damage. It is important to note that damage patterns and the amount of damage per impact scatter considerably.

Figure 2 shows the remaining damage after an impact with $\xi=-3$. In this violent event about 100 adatoms and 142 vacancies were created. Vacancy production in lower layers is well visible. The simulations also allow us to estimate the surface area influenced by a single step edge impact (the extension of the damage caused by the thermal spike). For the case visualized in Fig. 2 , it has a diameter of $d_{S} \approx 50 \AA$, corresponding to an area of about 300 surface atoms. It coincides quite well with the area delineated by the adatom rim. The extension of the central vacancy core is smaller and extends only to about $0.5 d_{S}$.

For the values $\vartheta=83^{\circ}$ under concern here, the interaction of the ions with the terrace may be described as planar surface channeling. ${ }^{43}$ The ions experience an effective planar surface potential and are nearly specularly reflected by its repulsive part. Whether or not surface damage is created during this interaction depends critically on the forces exerted by the ion onto the surface atoms during its motion. The experimental sputtering yield of $Y^{\text {terr }} \approx 0.08$ indicates nonnegligible damage on the terrace (due to the experimental beam divergence this value is an average over an angular spread of about $\pm 1.5^{\circ}$ ). As the adatom yield is typically two to four times larger than the sputtering yield, the frequent production of adatom surface vacancy pairs may be assumed. At $0 \mathrm{~K}, \mathrm{MD}$ simulations of terrace impacts with $\vartheta=83^{\circ}$ 


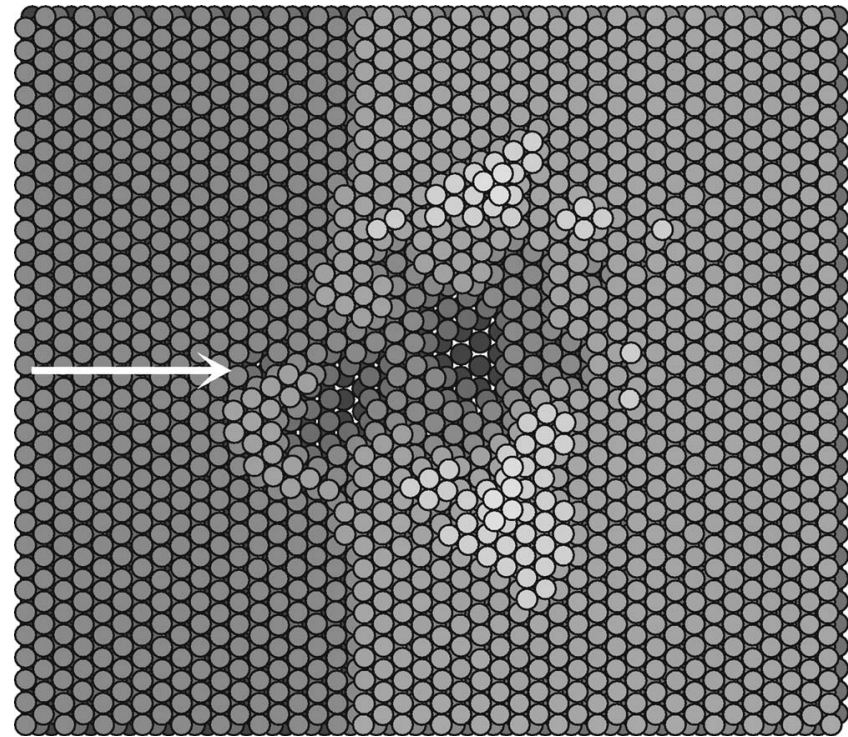

FIG. 2. Top view of the damage pattern of a $5 \mathrm{keV} \mathrm{Ar}^{+}$ion incident on the step on $\mathrm{Pt}(111)$ from the left with $\xi=-3$ (see the text). The arrow indicates the direction of the incoming ion.

show no damage production. Sputtering ceases for $\vartheta>79^{\circ}$ and adatom production for $\vartheta>81^{\circ}$. For finite temperatures, however, the situation changes. Due to surface atom vibrations the planarity of the surface potential is disturbed and larger forces are acting on individual surface atoms. In the MD simulations for $\vartheta=83^{\circ}$ at $550 \mathrm{~K}$, the adatom production is always present, though the yield depends critically on the simulation details, ranging from 0.04 to 0.55 . In conclusion, terrace impacts cause the creation of single adatoms and single surface vacancies with temperature dependent yields. The surface area influenced by a single terrace impact (the extension of the surface area heated by the ion collision) is far smaller for a terrace impact than for a step edge impact. From the molecular-dynamics simulations, we can derive that the heated area is not larger than about $130 \AA^{2}$ and contains about 20 atoms; the temperature increase reached there amounts at least to $250 \mathrm{~K}$ and assumes a maximum value of $600 \mathrm{~K}$.

In order to evaluate the importance of diffusion processes during pattern formation the frequency of their occurrence $\nu_{d}=1 / \tau_{d}$ has to be compared to the frequency $\nu_{e}=1 / \tau_{e}$, with which a surface site is influenced by the interaction with an impinging ion. The latter sets the relevant time scale $\tau_{e}$ of the experiment. Given the ion flux of $1.2 \times 10^{-3} \mathrm{MLE} / \mathrm{s}$ and assuming a step impact influenced area with a diameter of about $50 \AA$, steps illuminated by the ion beam and the stripes with a width of about $25 \AA$ adjacent to the steps are affected approximately every $3 \mathrm{~s}$ by a step edge impact $\left(\tau_{e} \approx 3 \mathrm{~s}\right)$. For all other surface locations, $\tau_{e}$ is much larger and each surface atom is affected roughly every $40 \mathrm{~s}$. With increasing temperature, a diffusion process must be considered to become relevant, whenever the condition $\tau_{d} \leqslant \tau_{e}$ becomes valid.

Table I groups the relevant $\mathrm{Pt}$ diffusion processes into bulk, surface point defect and cluster, step, and step edge barrier processes, and displays their onset temperatures $T_{0}$,

TABLE I. Diffusion processes relevant for pattern formation by ion bombardment. The temperatures given are estimates for the onset temperatures $T_{0}$ at which the frequency of a process is $1 \mathrm{~s}^{-1}$ (also see the text).

\begin{tabular}{|c|c|c|c|}
\hline & Process & $T_{0}$ & Reference \\
\hline \multirow[t]{3}{*}{ Bulk } & Interstitial atom diffusion & $20 \mathrm{~K}$ & 45 and 46 \\
\hline & Bulk vacancy diffusion & $500 \mathrm{~K}$ & 45 \\
\hline & $\begin{array}{l}\text { Bulk vacancy cluster } \\
\text { diffusion/dissolution }\end{array}$ & $500-850 \mathrm{~K}$ & 45 and 47 \\
\hline \multirow[t]{4}{*}{ Surface } & Adatom diffusion & $100 \mathrm{~K}$ & 48 and 49 \\
\hline & $\begin{array}{l}\text { Adatom cluster diffusion } \\
\text { (sizes } 2-7 \text { atoms) }\end{array}$ & $160-390 \mathrm{~K}$ & 50 \\
\hline & Surface vacancy diffusion & $\approx 180 \mathrm{~K}$ & 51 \\
\hline & Surface vacancy cluster diffusion & unknown & \\
\hline \multirow[t]{4}{*}{ Step } & $\begin{array}{l}\text { Mobility of laterally } \\
\text { onefold coordinated edge atoms }\end{array}$ & $\approx 150 \mathrm{~K}$ & 52 \\
\hline & $\begin{array}{l}\text { Mobility of laterally } \\
\text { twofold coordinated edge atoms } \\
\text { (step adatom diffusion) }\end{array}$ & $250-350 \mathrm{~K}$ & 53 and 54 \\
\hline & $\begin{array}{l}\text { Step adatom generation at kinks } \\
\text { (full edge mobility) }\end{array}$ & $500 \mathrm{~K}$ & $55-57$ \\
\hline & $\begin{array}{l}\text { Detachment of atoms from kinks, } \\
\text { (adatom lattice gas formation) }\end{array}$ & $700 \mathrm{~K}$ & 13,58 , and 59 \\
\hline \multirow[t]{2}{*}{ Step edge barrier } & $\begin{array}{l}\text { Insignificant for adatoms descending } \\
\text { at open (rough) steps }\end{array}$ & & 60 and 61 \\
\hline & $\begin{array}{l}\text { Significant for vacancies } \\
\text { at ascending steps }\end{array}$ & & 13,18 , and 58 \\
\hline
\end{tabular}



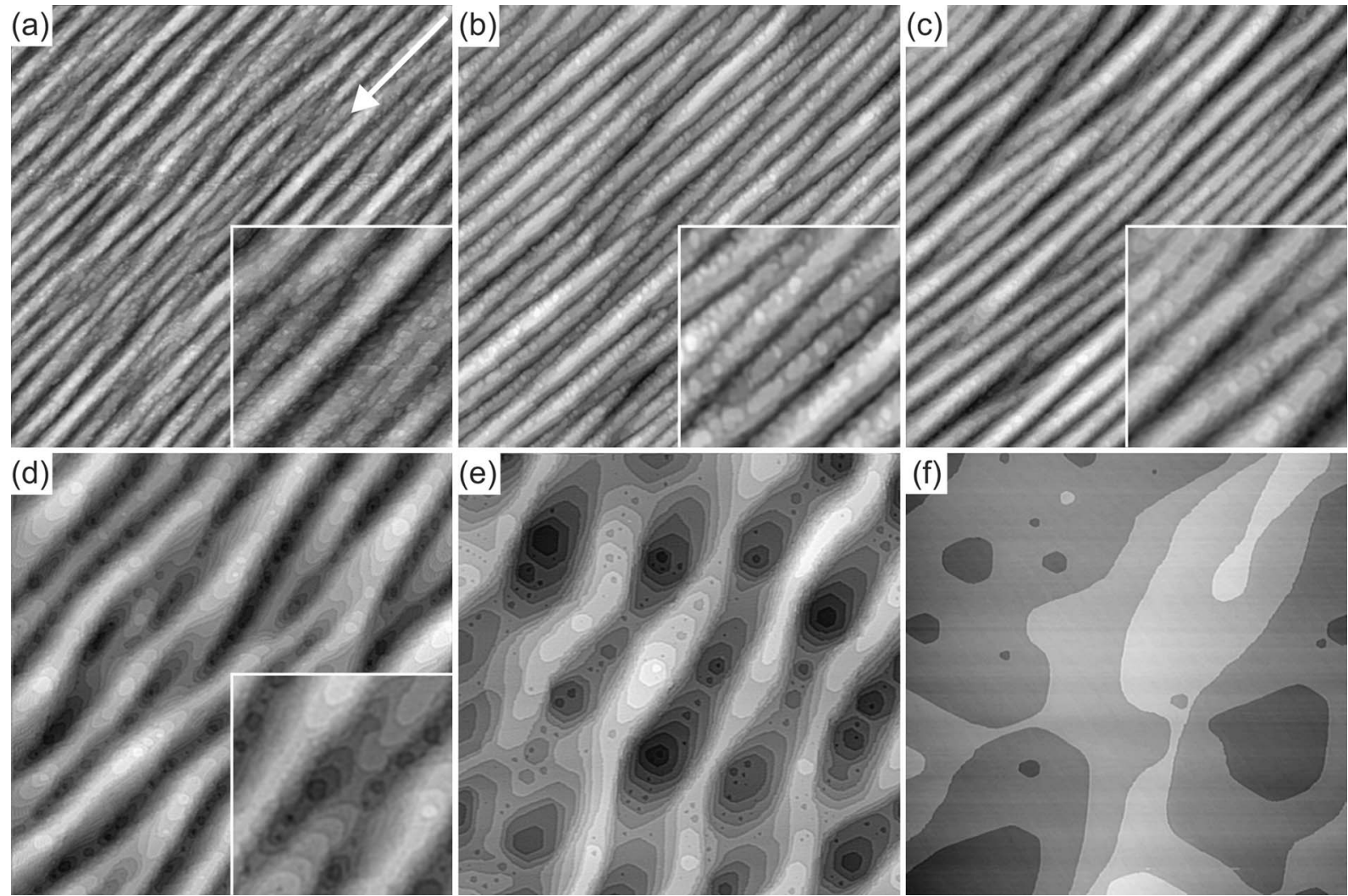

FIG. 3. Morphology of Pt(111) after an ion fluence of $20 \mathrm{MLE}$ at (a) $250 \mathrm{~K}$, (b) $350 \mathrm{~K}$, (c) $450 \mathrm{~K}$, (d) $550 \mathrm{~K}$, (e) $625 \mathrm{~K}$, and (f) $720 \mathrm{~K}$. The direction of the ion beam projection onto the surface is indicated by a white arrow in (a). The image size is $2450 \AA \times 2450 \AA$ for (a)-(e) and $4900 \AA \times 4900 \AA$ in (f). The insets show parts of the images in doubled magnification. The gray scale-height relation differs from image to image.

for simplicity for the usual time scale $\tau_{0}=1 \mathrm{~s} .{ }^{44}$ Below, two important morphological changes in the evolution of patterns will be recognized, which are linked to single atomic processes. The first significant step is the onset of full step edge mobility at $500 \mathrm{~K}$, i.e., the transport of material along the step edge, even in the absence of ion bombardment. Full edge mobility requires the creation of step adatoms at kink positions (laterally threefold coordinated kink atoms become laterally twofold coordinated step adatoms) and the motion of these mobile species along the step edge, even around corners. The second is the formation of an adatom lattice gas at $700 \mathrm{~K}$ by the detachment of atoms from kink positions, thereby becoming adatoms, and their migration onto the terrace. The latter process is relevant, as it bypasses effectively the step edge barrier for vacancies, i.e., the kinetic hindrance for surface vacancies to anneal at ascending steps. ${ }^{13,59}$

A remark with respect to the step edge barrier for adatoms is in place here, which is frequently discussed as a destabilization mechanism in ion bombardment. ${ }^{8,31}$ For dense packed straight step edges oriented along the $\langle 110\rangle$ directions, Pt adatoms on $\mathrm{Pt}(111)$ experience a significant additional step edge barrier against their descent to a lower layer of up to $0.20 \mathrm{eV} .^{60}$ This barrier is indeed relevant in mound growth experiments at 400-500 K, where such steps form due to the step edge diffusion kinetics. For open, kinked steps, the step edge barrier amounts-depending on the kink concentration-only to a few hundreds of an eV. In grazing- incidence ion bombardment along the [1-112] direction the formation of open kinked steps along this direction is forced by the ion beam. Thus, the additional step edge barrier for adatoms is irrelevant for the morphological evolution under the conditions used here. It is therefore not discussed any further.

As a concluding remark to this section, the applicability of continuum theory approaches to the present case is addressed. One of the essentials in continuum theory is the smooth and continuous variation of the local sputtering yield with the surface morphology. This assumption is violated to the extreme in the present case, where a nearly digital variation of the sputtering yield from terraces to steps takes place, a variation by a factor of about 100 . It is therefore no surprise that none of the global predictions of the BradleyHarper theory for pattern formation is fulfilled in the present case: roughness neither grows exponentially (not even in an initial stage) nor does the pattern wavelength stay constant with ion fluence. As the continuum theory is inapplicable, it is not addressed more in the following parts of the paper.

\section{THE TEMPERATURE DEPENDENCE OF RIPPLE FORMATION-OVERVIEW}

Figure 3 gives an overview on the temperature dependence of the surface morphology of $\mathrm{Pt}(111)$ after an ion flu- 

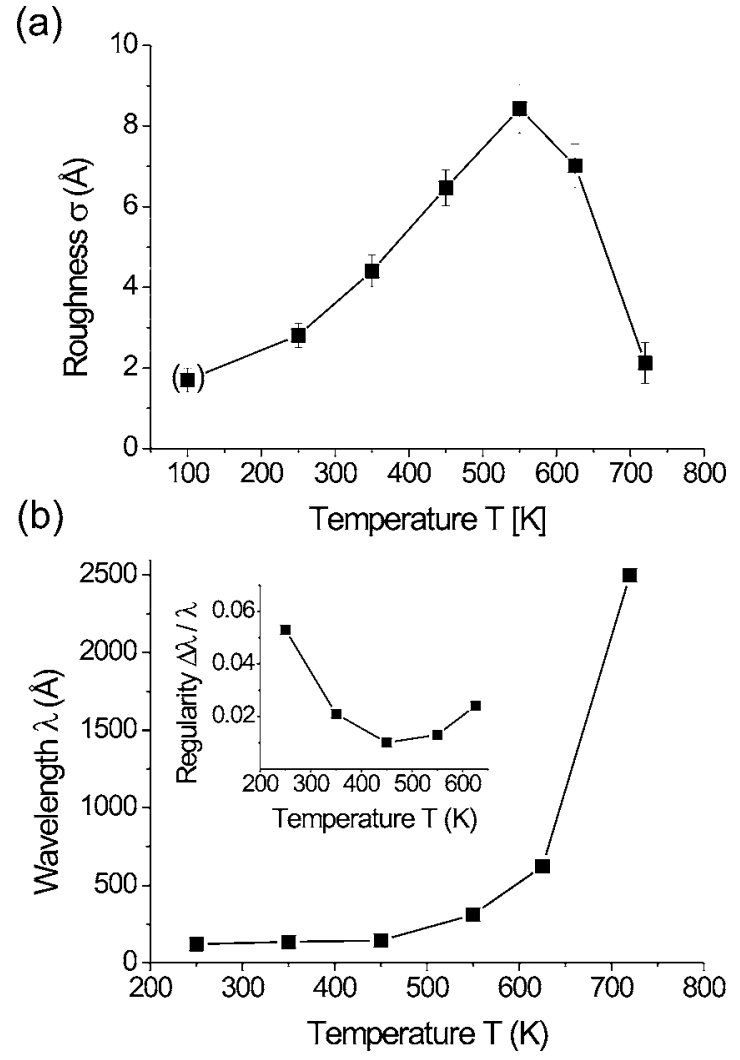

FIG. 4. (a) Temperature dependence of roughness $\sigma$ for the morphologies of Fig. 3 formed by exposure to ion fluences of 20 MLE. The roughness data point for $100 \mathrm{~K}$ after $70 \mathrm{MLE}$ added in brackets (compare Fig. 5). (b) Wavelength analysis for the same patterns. Error bars below the symbol size, except for the data point at $720 \mathrm{~K}$, which is just an estimate with a large uncertainty toward larger values. Inset: normalized standard deviations $\Delta \lambda / \lambda$ of the histograms of distances $\lambda$ between valleys in the direction normal to the ion beam versus temperature (see the text).

ence of 20 MLE. The following observations are obvious from the images: (i) For all but the highest temperature the formation of a ripple pattern along the direction of the ion beam projection onto the surface is observed. (ii) The typical height difference between the ripple ridges and the ripple valleys is of the order of ten atomic layers. In fact, the quantitative roughness analysis in Fig. 4(a) exhibits a bell shaped roughness curve with a maximum in roughness around $550 \mathrm{~K}$. The roughness is much smaller at low and at high temperatures. A similar bell shaped temperature dependent roughness evolution was observed also for the normal incidence ion bombardment of $\operatorname{Ag}(001)^{14}$ and $\operatorname{Ag}(110) .{ }^{62}$ (iii) The step edge structure changes with increasing temperature (compare insets of Fig. 3). The step edges become smoother and the visible adatom islands in the top layer become larger and more compact. Moreover, above $450 \mathrm{~K}$ the global step orientation in the ripple pattern starts to deviate more and more from the $[\overline{1} \overline{1} 2]$ direction of the ion beam. Portions of dense packed $\langle 110\rangle$ steps develop. (iv) While for low temperatures only narrow V-shaped valleys are visible, at temperatures above $450 \mathrm{~K}$ broad U-shaped valleys are formed. At the bottom of the $\mathrm{V}$ valleys only narrow vacancy grooves are visible, while at the bottom of the U-shaped valleys compact vacancy islands are present, which are bounded by the energetically favorable $\langle 110\rangle$ oriented steps. (v) With increasing temperature the pattern changes from being dominated by broad ripple ridges (separated by $\mathrm{V}$-shaped valleys) to one being dominated by broad U-shaped ripple valleys (separated by comparatively narrow ridges). The difference between the ripple ridge and the ripple valley pattern is most obvious in the comparison of Fig. 3(b) and Fig. 3(e). This change in the character of the ripple pattern with temperature is similar to the observed change from dot to pit patterns at normal incidence ion bombardment. ${ }^{10,12,20}$ (vi) Finally, the ripple wavelength apparently stays roughly constant up to $450 \mathrm{~K}$ and then rapidly increases with temperature. This finding is corroborated by the quantitative analysis of Fig. 4(b), where the ripple wavelength is plotted versus temperature. Up to $450 \mathrm{~K}$ the wavelength stays around $120 \AA$ and then increases rapidly. The data point at $720 \mathrm{~K}$, where after an ion fluence of 20 MLE only the anticipation of a ripple pattern is visible, is an estimate with considerable uncertainty. We note that a similar dependence of ripple wavelength on temperature was found for the nanogrooves on $\mathrm{Cu}(001) .{ }^{34}$ (vii) The regularity of the ripple pattern in the direction normal to the ion beam, i.e., the uniformity of the wavelength is best developed at $450 \mathrm{~K}$. It is quantitatively measured from the relative standard deviations $\Delta \lambda / \lambda$ of the histograms of distances $\lambda$ between valleys in the direction normal to the ion beam [compare the inset of Fig. 4(b)].

The previous observations advise a distinction of two regimes of ripple formation. The athermal regime with a temperature independent wavelength between 200 and $500 \mathrm{~K}$ and the thermal regime between 500 and $700 \mathrm{~K}$. As discussed previously, the two regimes of pattern formation differ not only by their different dependence of wavelength on temperature, but also by their different appearance (ripple ridge versus ripple valley pattern, transition from $\mathrm{V}$-shaped valleys to U-shaped valleys), the degree of alignment of the observed steps with respect to the ion beam direction, as well as the presence/absence of compact vacancy islands. The two regimes of pattern formation are limited toward higher and lower temperatures by the absence of pattern formation. The absence of pattern formation above $700 \mathrm{~K}$ is obvious from Fig. 3(f). The absence of pattern formation at low temperature can be inferred from Fig. 5. It exhibits an STM topograph taken after exposure to $70 \mathrm{MLE}$ at $100 \mathrm{~K}$. Although a faint overall alignment of the surface structures along the ion beam direction (indicated by the arrow) is visible, it would be a clear exaggeration to talk about a pattern. The topography is dominated by numerous small adatom clusters. Only a few layers are uncovered and the roughness is well below the value obtained at $250 \mathrm{~K}$ after exposure to $20 \mathrm{MLE}$ [compare Fig. 3(a)].

\section{THE ATHERMAL PATTERN FORMATION REGIME}

The key to uncovering the mechanisms of pattern formation is an analysis of its onset. Figure 6 exhibits a sequence of STM topographs representing the morphology at $450 \mathrm{~K}$ after ion fluences decreasing from the 20 MLE shown in Fig. 


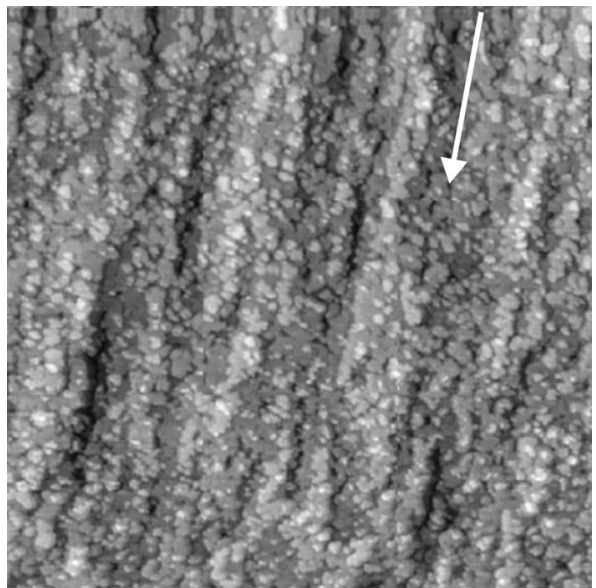

FIG. 5. Morphology of Pt(111) after an ion fluence of 70 MLE at $100 \mathrm{~K}$. The direction of the ion beam projection onto the surface is indicated by the white arrow. The image size is $1250 \AA$ $\times 1250 \AA$.

3(c) toward lower values. In Fig. 6(a) after an ion fluence of only 5 MLE, a fully developed ripple pattern with ripple ridges separated by narrow, several layers deep V-shaped valleys is present. Decreasing the ion fluence further, in Fig. 6(b) after 1 MLE, a periodic pattern on the terrace is still present. It consists of elongated adatom island structures (in the following also named adatom bands) remaining from the first, half removed layer. Alternatively phrased, the pattern is formed by a network of coalesced elongated vacancy islands (or vacancy grooves). Even in Fig. 6(c) after an ion fluence of only 0.5 MLE, a periodicity is visible. The topograph exhibits a preexisting step (present already before the start of ion exposure) crossing the topograph horizontally at about half image height. The ion exposure generated periodic vacancy fjords at this step prior to the appearance of the pattern on the terrace. The periodicity of the vacancy fjord pattern of Fig. 6(c) in the direction normal to the ion beam is identical to the one of the terrace vacancy groove pattern of Fig. 6(b) within the limits of error and amounts to $75 \AA$. Besides the vacancy fjords at the preexisting step, on the terrace vacancy grooves, small compact vacancy islands and adatom islands are visible in Fig. 6(c).
We focus first on the formation of the pattern on the terrace and distinguish - as suggested by the topographs of Fig. 6-two stages: (I) The formation of long vacancy island structures-vacancy grooves-along the ion beam direction and (II) their periodic arrangement in the direction normal to the ion beam. According to the MD simulations, although terrace impacts cause no or only negligible sputtering occasionally they create vacancies and adatoms. These defects will partly recombine, and partly migrate at the temperatures under concern and form larger vacancy and atom clusters. The cluster steps are illuminated by the ion beam and will therefore cause step impacts. Step impacts cause the formation of large vacancy clusters (or small vacancy islands) surrounded by adatoms. The adatoms will largely recombine with the vacancy islands by diffusion, without being able to fill them due to the sputtered material. Ensuing step impacts will cause a rapid extension of the vacancy islands in the direction of the ion beam. As long as step edge diffusion is unable to reshape the vacancy islands, they remain narrow and elongated and eventually form the vacancy grooves, as apparent in Fig. 6(c). This condition is fulfilled as long as no step adatom generation at kinks is possible, which in turn would cause full step edge mobility (compare Table I). The motion of onefold or twofold coordinated step atoms as well as adatoms filling in will certainly smoothen the ensuing vacancy groove step, but does not allow transport along the kinked [ $\overline{1} \overline{1} 2]$ direction, the direction along which the ion beam creates new step edge.

To understand stage (II) of pattern formation, i.e., the development of a periodicity of the vacancy grooves normal to the beam direction, the separation $d$ of vacancy grooves normal to their growth direction has to be to compared to the diameter $d_{S}$ of the area influenced by a single step edge impact defined in Sec. III. As shown in Fig. 7(a), if $d>d_{S}$, two vacancy grooves will pass by each other without any interaction. If $d \approx d_{S}$ [compare Fig. 7(b)], a vacancy groove tip approaching the end of another vacancy groove will influence its position. The melt outflow of a thermal spike created at the tip of the vacancy groove in consequence of a step impact will partly fill the neighboring vacancy groove and tend to shift its center away from it. This mechanism creates an effective repulsion for vacancy grooves. Finally, as sketched in Fig. 7(c), if $d<0.5 d_{S}$ the growing tip of the
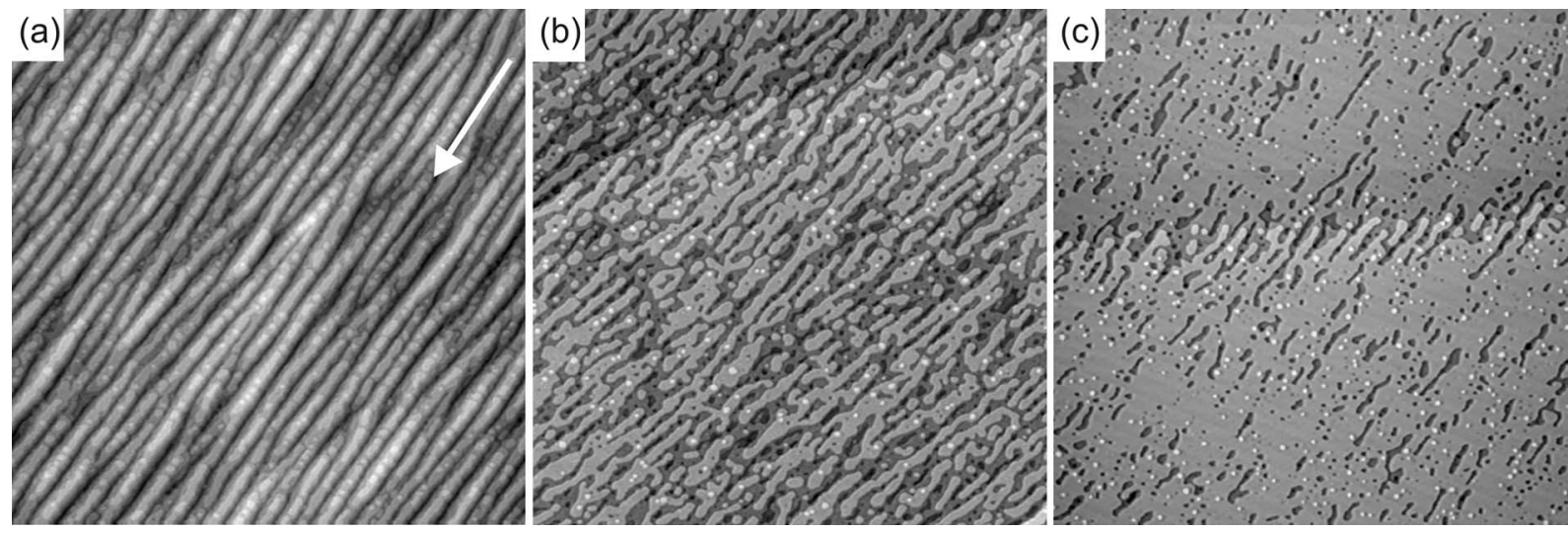

FIG. 6. The sequence of topographs after ion bombardment at $450 \mathrm{~K}$ with decreasing ion fluence. (a) $5 \mathrm{MLE}$, (b) 1.0 MLE, (c) 0.5 MLE. The image size is always $2450 \AA \times 2450 \AA$. The white arrow indicates the direction of the ion beam. 


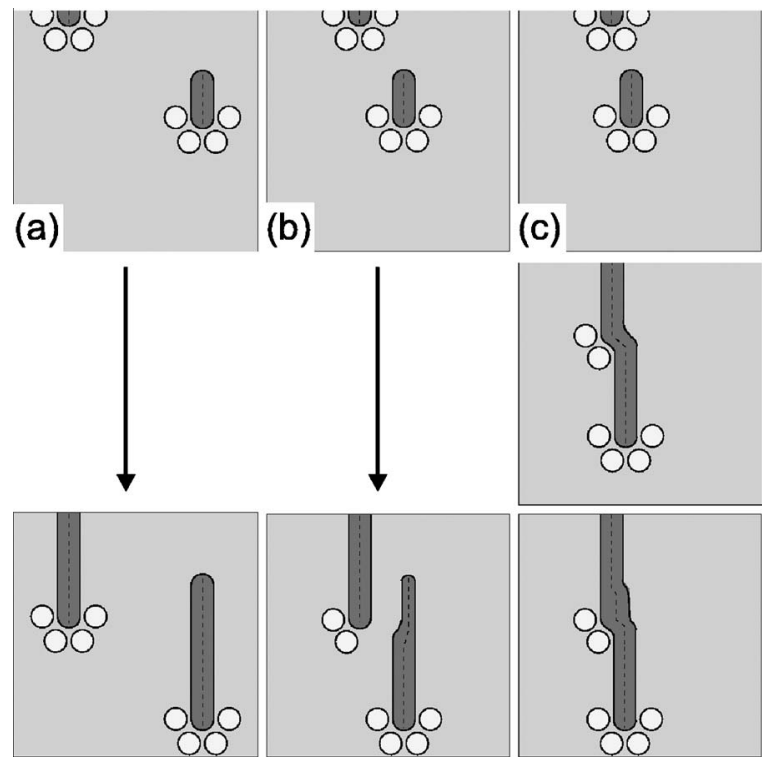

FIG. 7. Cartoons illustrating the different scenarios for the interaction of growing, elongated vacancy islands. (a) Vacancy groove separation normal to the beam $d>d_{S}$ : no interaction. (b) $d$ $\approx d_{S}$ : repulsion (see the text). (c) $d<0.5 d_{S}$ : coalescence.

vacancy groove will hit the end of another one, inducing vacancy groove coalescence. If there is a certain misalignment between the two grooves, upon coalescence an illuminated piece of step is formed. As indicated in Fig. 7(c), this kink will be preferentially eroded by the ion beam. Moreover, due to adatom outflow the neighboring step edge will be shifted, thereby improving the overall alignment.

In this somewhat speculative scenario the initial pattern wavelength $\lambda$ is set by the ion beam, more precisely by the impact separation $d_{S}$. The rough agreement of the initial pattern wavelength $\lambda \approx 75 \AA$ with $d_{S} \approx 50 \AA$ supports our speculations. If the initial $\lambda$ is set by the ion beam, it is natural that there exists an extended temperature range, in which $\lambda$ is independent of temperature, as observed experimentally. For the same reason, $\lambda$ is independent of ion flux up to $450 \mathrm{~K}$. Since $d_{S}$ is an increasing function of the ion energy, one expects an increase of the $\lambda$ with the ion energy. Indeed, for a fixed ion fluence we observe for $2 \mathrm{keV} \mathrm{Ar}^{+}$a wavelength smaller by $40 \%$ compared to $10 \mathrm{keV} \mathrm{Ar}^{+} .{ }^{35} \mathrm{Fi}-$ nally, it is nothing but expected that the same periodicity is observed to develop on terraces and at preexisting step edges, if the initial $\lambda$ is set by the ion beam. In fact, basically the same mechanisms as sketched previously and illustrated in Fig. 7 hold also for the early pattern formation at preexisting step edges. This step edge pattern is formed more rapidly, as the illuminated step edges are present right from the beginning of ion exposure, while on the terrace the illuminated cluster steps are formed only after an induction phase. The duration of the induction phase is related to the amount of damage caused by terrace impacts. Thus, decreasing the angle of incidence by a few degrees - thereby increasing the perpendicular kinetic energy and thus the terrace damageshortens the induction phase (compare Ref. 36).

If the initial pattern wavelength is set by the ion beam, one may wonder why then pattern formation diminishes be- low $200 \mathrm{~K}$, as demonstrated by Fig. 5. Apparently some diffusion processes are necessary for pattern formation. A look into Table I indicates that below $200 \mathrm{~K}$ the mobility of vacancies, small adatom clusters, and adatoms ceases. In fact, their mobility is decisive in the initial stage of pattern formation for the following two reasons. In the space between two vacancy grooves, i.e., on the adatom band separating them, terrace impacts still create vacancies and adatoms. Diffusion warrants that these vacancies and adatoms diffuse to existing sinks, namely to the steps separating adatom bands and vacancy grooves (depletion effect). In the absence of diffusion, these vacancies and adatoms remain at their location and initiate the formation of new vacancy grooves on an adatom band "too small," i.e., with a linear dimension below $d_{S}$. Their formation destroys the ordering of the vacancy grooves and thus disturbs the pattern formation. Moreover, in the absence of vacancy, adatom cluster, and adatom diffusion, also the formation of vacancy grooves themselves is hampered. The damage reorganization and partial healing after step edge impacts is crucial to ensuring the evolution of a single, elongated vacancy island.

One may rephrase and generalize these arguments by stating that in the absence of vacancy, adatom cluster, and adatom diffusion, the formation of vacancy grooves is initiated at too many locations and at too many times, thus burying the pattern formation in noise. In fact, in Figs. 8(a) and 8(b), it is seen that the initial stage of pattern formation becomes more noisy toward lower temperatures before it eventually ceases.

The pattern formation mechanism presented here relies on the presence of steps illuminated by the ion beam, be it in the form of preexisting steps or of the vacancy or adatom clusters on the terrace. Nucleation is irrelevant for pattern formation: elongated vacancy islands can grow out of a single vacancy or adatom on the terrace. Vacancy, adatom, and small adatom cluster mobility is, however, essential for pattern formation as it limits the number of places of vacancy island formation and suppresses new vacancy groove formation after the onset of pattern formation.

\section{THE THERMAL PATTERN FORMATION REGIME}

In distinction to the athermal pattern formation regime, in the thermal pattern formation regime the developing wavelength is strongly temperature and also ion flux dependent. The origin of the onset of wavelength increase is most easily identified by comparing the morphologies after low ion fluence. Figure 8 exhibits STM topographs after 0.5 MLE ion exposure at temperatures from 250 to $720 \mathrm{~K}$. Up to $450 \mathrm{~K}$ predominantly vacancy groove structures aligned along the ion beam evolve, while above $450 \mathrm{~K}$ the picture is qualitatively different: the visible vacancy island structures are compact, exhibiting the energetically preferred and densepacked $\langle 110\rangle$ steps. Apparently, at $550 \mathrm{~K}$ and above the full step edge mobility (see Table I) is present and keeps the vacancy island close to its minimum energy shape, despite the anisotropic removal of material by the ion beam. As growth of a vacancy island structure is not more limited to the ion beam direction (as in the athermal pattern formation 

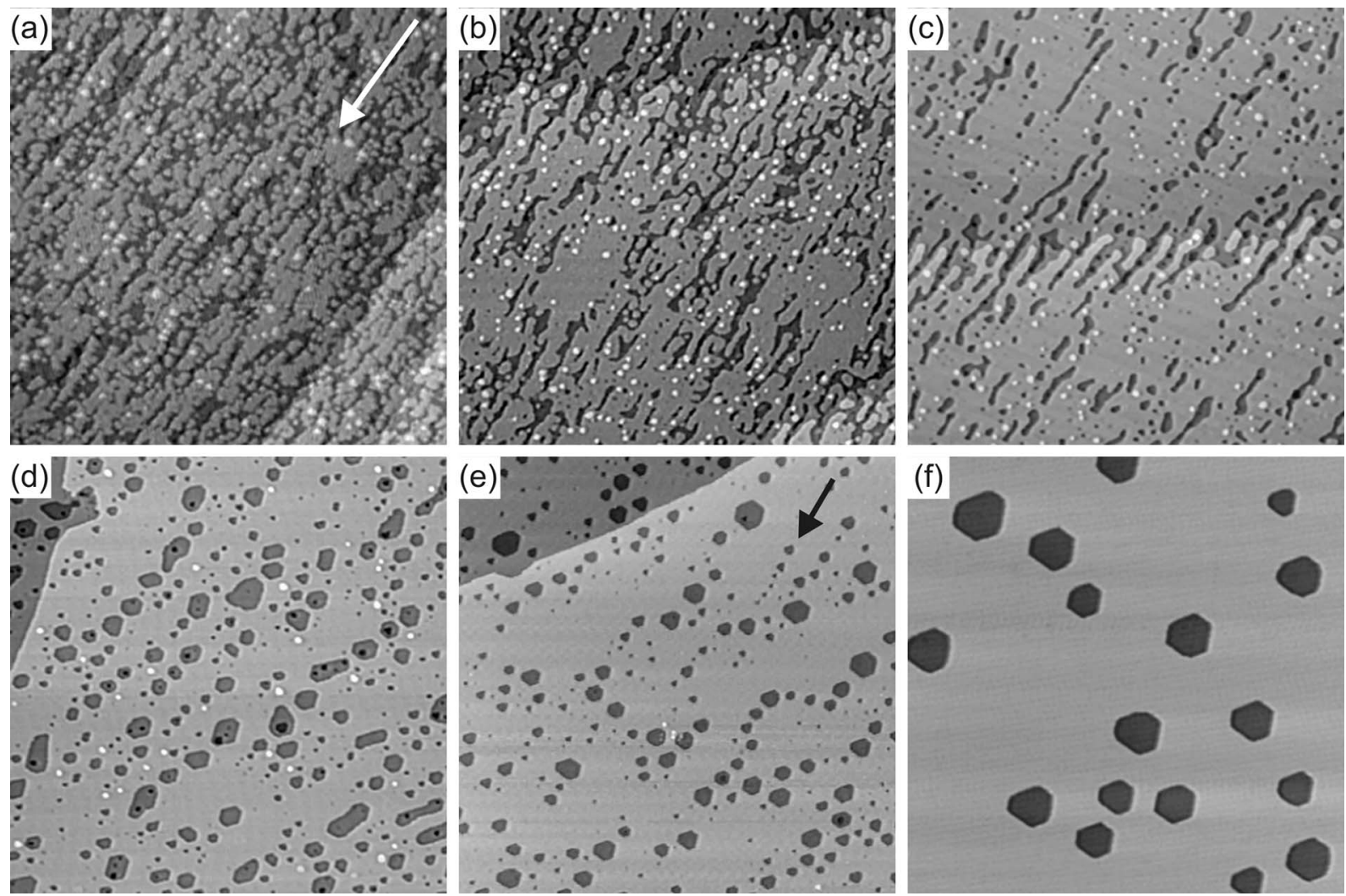

FIG. 8. Morphology of Pt(111) after an ion fluence of $0.5 \mathrm{MLE}$ at (a) $250 \mathrm{~K}$, (b) $350 \mathrm{~K}$, (c) $450 \mathrm{~K}$, (d) $550 \mathrm{~K}$, (e) $625 \mathrm{~K}$, and (f) $720 \mathrm{~K}$. The direction of the ion beam projection onto the surface is indicated by a white arrow in (a). The black arrow in (e) indicates a chain of vacancy islands with positional order. The image size is $1650 \AA \times 1650 \AA$ for all topographs.

regime), but is isotropic, vacancy islands reach a much larger extension normal to the beam direction. This increase implies a larger length scale in the direction normal to the beam in the thermal pattern formation regime and thus a larger wavelength. ${ }^{63}$

Nevertheless, it is not straightforward to imagine how under these conditions of isotropic vacancy island growth a ripple pattern reflecting the bombardment geometry may result at all. The solution to this puzzle is visible in Fig. 8. A close inspection of Figs. 8(d) and 8(e) reveals a considerable amount of positional order of the compact vacancy islands. The order is most easily recognized by looking to these images under a grazing observation angle from various directions. The vacancy islands are partly aligned along the ion beam direction. A nice example for this alignment is highlighted by the black arrow in Fig. 8(e).

As discussed in our recent Letter, ${ }^{36}$ ions entering the space between the surface layer and the layer below at an ascending step may be guided for substantial distances between these lattice planes until vibrations eventually cause a large angle scattering event and dechanneling. At the location of dechanneling, which may be located more than $100 \AA$ behind the entering step edge, considerable damage results in the form of large vacancy and adatom clusters. Subsequent step edge impacts into these cluster steps will cause the formation of a new independent vacancy island. Assuming the step, where the channeling ion initially entered, to be the ascending boundary of a mother vacancy island, this process causes the formation of a daughter vacancy island. With respect to the ion beam direction, the daughter vacancy island is found behind the mother vacancy island due to its origin from the dechanneling damage. By iteration, this mechanism leads to chains of vacancy islands aligned in the ion beam direction, as visible in Figs. 8(d) and 8(e). We note that the damage created on the channeling path has, in principle, also the chance to cause step edge impacts. Consequently, two or more daughter islands may originate from a single channeling event, although the probability for this case is presumably small.

With the help of this information it is now straightforward to explain stage (I) of pattern formation in the thermal regime, i.e., the formation of an island groove. Upon island growth, the positional alignment of compact vacancy islands causes their preferential coalescence along the ion beam direction. The resulting island grooves are not transformed into compact islands for two reasons: First, their size (step edge length) increases suddenly upon coalescence, making reshaping of the island slow on the experimental time scale. The reshaping time $\tau$ increases as $\tau \propto d^{\alpha}$, where $d$ is the final, compact island diameter. For edge diffusion $\alpha=4$ is expected, ${ }^{64,65}$ in good agreement with experiments. ${ }^{66,67} \mathrm{Sec}-$ ond, as visible in the MD simulations (compare Fig. 2), step edge impacts frequently cause not only the removal of atoms from the first layer, but also the creation of vacancies in the second or even lower layers. Thus, already at the time of coalescence many of the compact first layer vacancy islands 


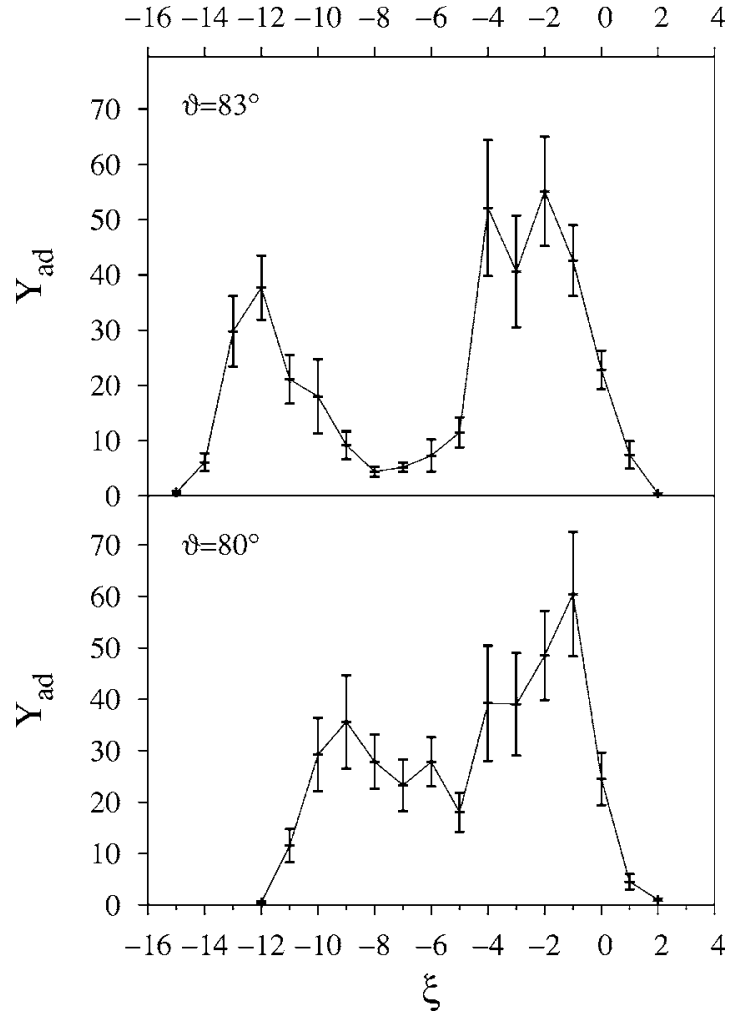

FIG. 9. A comparison of the adatom yields for ions impinging with $80^{\circ}$ and with $83^{\circ}$ toward the target normal. Data show the dependence of the adatom yield calculated by molecular dynamics as a function of the distance from the step location. $\xi=x / \Delta x$ measures the distance of the ion impact point to the step edge in units of the spacing of dense packed atomic rows on the (111) surface.

contain second layer vacancy islands [compare Fig. 6(b) and Fig. 8(d)]. These second layer vacancy islands impede the motion of the coalesced island groove step by step-step repulsion, ${ }^{68}$ thereby stabilizing its shape. It should be noted that planar subsurface channeling takes place also in the athermal pattern formation regime [compare Fig. 8(c)], but it is not of significant relevance for vacancy groove formation. Aligned vacancy cluster formation is unimportant due to the fast motion of illuminated vacancy groove steps in the ion beam direction (about $30 \mathrm{~nm}$ for each MLE of fluence for $\vartheta=83^{\circ}$ ).

A proof for the importance of planar subsurface channeling to pattern formation in the thermal regime is obtained by performing experiments in which the effect of channeling is switched off. Figure 9 compares the adatom yields obtained by molecular-dynamics simulation under $83^{\circ}$ and under $80^{\circ}$ impact angle towards the target normal. ${ }^{69}$ The data for $83^{\circ}$ impact show a pronounced dip at $\xi \cong-7$, which has its origin in the channeling of the projectile: ${ }^{39}$ When the ion impinges on the lower terrace at this location, it is specularly reflected and enters the upper terrace just in between the first and the second monolayer, where it can be channeled and consequently delivers only a relatively small amount of energy in the vicinity of the step to the target, inducing there only little sputtering and adatom production. This "channeling dip" is largely absent for $80^{\circ}$ impact angle, proving that in this case, the probability for channeling is largely reduced.
For the initial stages of pattern formation (ion fluences up to $2.0 \mathrm{MLE}$ ), we showed in Ref. 36 that the removal of planar subsurface channeling by a reduction from $\vartheta=83^{\circ}$ to $\vartheta=79^{\circ}$ largely diminishes ordering of the early stage pattern and its alignment with respect to the ion beam direction. Without channeling, the aligned formation of vacancy islands is absent, giving rise to a nearly isotropic labyrinth pattern at the moment of coalescence of the first layer vacancy islands. Nevertheless, even in the absence of channeling some alignment of the pattern with respect to the ion takes place during subsequent growth. The remainders in the upper layers are unable to reshape due to pinning by lower layer steps. As the ion beam causes a global motion of all illuminated steps in the beam direction, the remainder structures extending normal to the beam will be removed first (small thickness normal to the beam) while the parts in the beam direction survive longer (large thickness normal to the beam). The further the erosion progresses and the larger the amplitude of the pattern, the more relevant this effect will become (all but the lowest layers are remainder layers). Thus, one might speculate that the better alignment of the pattern due to channeling is only an initial state effect that completely disappears after sufficiently long bombardment. Figure 10 exemplifies that this speculation is wrong. In Fig. 10 (a), a quite irregular pattern evolves at $550 \mathrm{~K}$ for $\vartheta=79^{\circ}$ after 14 MLE. The pattern is predominantly of ripple or canyon character, with a considerable mound/pit component due to the significant roughness along the ion beam direction. The superior pattern order for $\vartheta=83^{\circ}$ is evident by comparison to Fig. 10(b), which exhibits the topography after the same removed amount. Thus, the aligned vacancy island formation by planar subsurface channeling is in this temperature regime necessary for the fabrication of well oriented patterns, irrespective of the erosion time.

We turn now to the discussion of the stage (II) of pattern formation in the thermal regime, i.e., to the ordering in the direction normal to the ion beam. The comparison of Fig. 1(e) of Ref. 36 (1.0 MLE at $550 \mathrm{~K}$ ) with Fig. 6(b) (1.0 MLE at $450 \mathrm{~K}$ ) makes obvious that the order of the vacancy island grooves in the direction normal to the ion beam at $550 \mathrm{~K}$ is worse than at $450 \mathrm{~K}$. Also, the inset of Fig. 4(b) shows a worsening in pattern regularity above $450 \mathrm{~K}$. Pattern evolution in the sense of an array of structures with a characteristic separation is thus hampered by the onset of full edge mobility. Moreover, the comparison makes two more differences visible: First, the vacancy grooves are due to their compact growth prior to coalescence much broader than at $450 \mathrm{~K}$. These broad vacancy grooves, which may be traced back to the onset of step edge mobility along vacancy island edges are the origin of the transition from a ripple ridge pattern to a ripple valley pattern (being dominated by broad U-shaped valleys) with increasing temperature, as observed in Fig. 3. Second, large top terrace spaces are left between vacancy grooves, which result from a more dephased vacancy groove formation, if compared to the situation at $450 \mathrm{~K}$. During subsequent ion exposure there is a significant probability for new vacancy island and subsequent vacancy groove formation in these larger terrace spaces, while the depletion effect mentioned in Sec. V will prevent the formation of new vacancy islands on narrow terrace spaces. Thus again, this ef- 

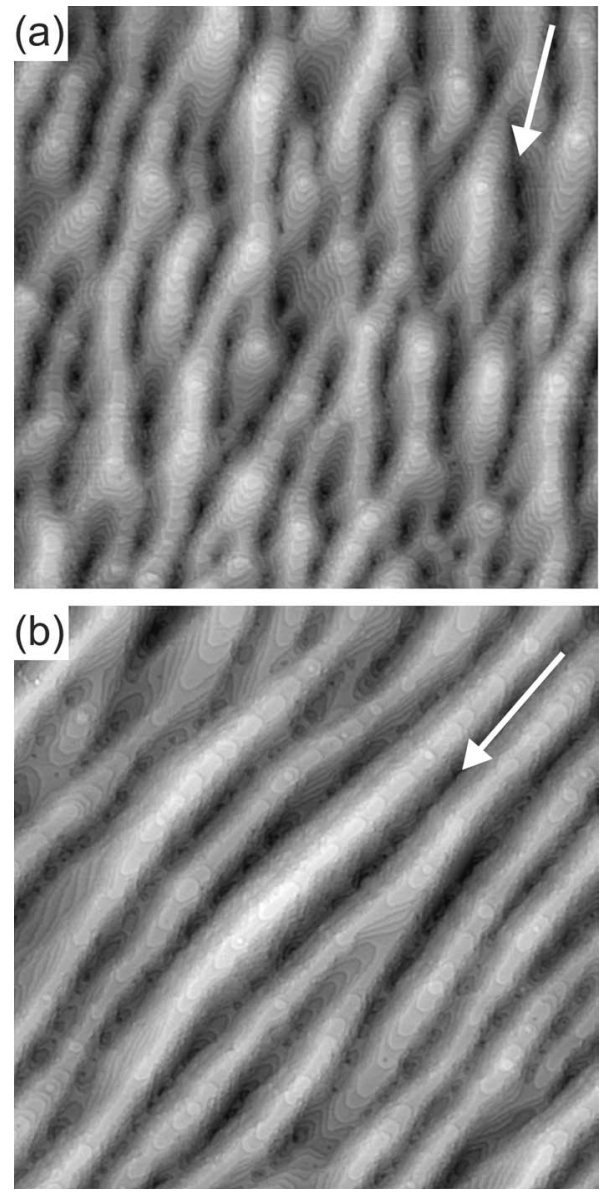

FIG. 10. Morphologies of $\mathrm{Pt}(111)$ after the removal of identical amounts of material. (a) $\vartheta=79^{\circ}, 550 \mathrm{~K}, 14 \mathrm{MLE} 5 \mathrm{keV} \mathrm{Ar}^{+}$. (b) $\vartheta=83^{\circ}, 550 \mathrm{~K}, 20 \mathrm{MLE} 5 \mathrm{keV} \mathrm{\textrm {Ar } ^ { + }}$ at $550 \mathrm{~K}$. The direction of the ion beam is indicated in each topograph by a white arrow. The image sizes are $2450 \AA \times 2450 \AA$.

fect is a first element contributing to vacancy groove ordering. As there is very little sputtering from the adatom bands separating the vacancy grooves, adatom and vacancies are produced in similar numbers and if not recombined immediately, arrive in similar numbers at the neighboring vacancy grooves. Therefore they have no or only a negligible effect on vacancy groove ordering.

With the help of Fig. 11 we evaluate now the importance of adatoms and vacancies created at the bottom of vacancy grooves. These adatoms and vacancies are created in significant numbers due to step edge impacts into second layer compact vacancy clusters, of which the origin has already been mentioned in the discussion of stage (I) previously. Figure 11 displays cartoons of surface cross sections in the direction normal to the ion beam with three vacancy grooves separated by two remaining top layer terraces. The center vacancy groove has a larger width than the neighboring two grooves. By step edge impacts, vacancies are removed from their bottom and adatoms are created (dotted changes in the upper panel). Even if single vacancies are created, they experience a significant step edge barrier at the ascending step and are thus attached to the existing second layer vacancy island. The majority of the adatoms, however, attach to the

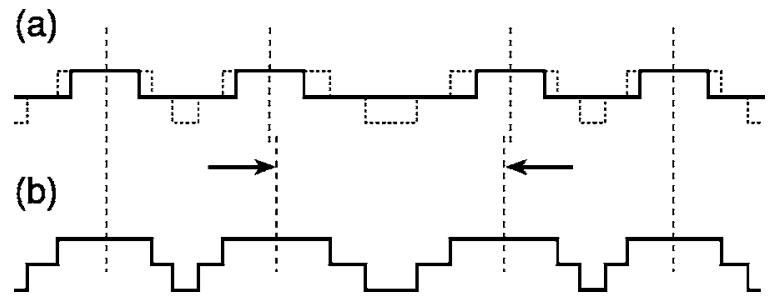

FIG. 11. Cartoons of surface cross sections in the direction normal to the ion beam with three vacancy grooves separated by two remaining top layer terraces. The center vacancy groove has a larger width than the neighboring ones. (a) Initial situation (full lines), centers of top layer terraces (dotted lines), and changes due to subsequent ion exposure (dashed lines). (b) The final situation (full lines) and centers of the remaining top, terraces (dashed lines). The lateral shift of these centers upon exposure is indicated by arrows.

nearby ascending step edges, while a minority may recombine with second layer vacancies. On the bottom of the central large vacancy groove in the average, more vacancies and adatoms will be created. In consequence, the width of the vacancy grooves becomes more similar. Most important, the average separation of the adatom band centers becomes more uniform, thereby improving the pattern periodicity. As shown in Fig. 1(f) of Ref. 36, after an ion fluence of 2.0 MLE (canyon depth of 2-3 ML) the erosion pattern is developed and in regularity comparable to the pattern after an ion fluence of 1.0 MLE at $450 \mathrm{~K}$ [compare to Fig. 6(b)].

To conclude the discussion of the thermal pattern formation regime, its termination toward high temperatures needs to be briefly discussed [compare Fig. 3(f)]. The investigation of normal incidence ion bombardment of $\mathrm{Pt}(111)$ identified the onset of atom detachment from kinks at $700 \mathrm{~K}$ (compare Table I) as the mechanism for the substantial decrease of roughness above $700 \mathrm{~K} \cdot{ }^{13,17,18,58,59}$ The detachment of atoms from steps causes the formation of an adatom lattice gas within the evolving compact vacancy islands. Thereby the nucleation and growth of vacancy clusters on their bottom is effectively suppressed. Free adatoms experience an only negligible step edge barrier when descending into a vacancy or vacancy island (compare Table I). The adatom lattice gas formation bypasses not only the step edge barrier for vacancies effectively, but also causes a rapid filling of vacancy clusters at their bottom that are created, e.g., by step edge impacts. Consequently, the vacancy islands grow large and coalesce prior to the formation of new vacancy islands at their bottom. Only the remainders of the coalescence processes-elongated adatom island structures-lead to a gradual and slow roughness buildup. ${ }^{59}$

Concluding this section, a comparison to the nanogroove formation scenario on $\mathrm{Cu}(001)$ by $0.8 \mathrm{keV} \mathrm{Ar}^{+}$grazing incidence $\left(\vartheta=80^{\circ}\right)$ ion bombardment is in place. ${ }^{34}$ Very similar to the present investigation, the authors report two regimes of pattern wavelength: one with a fixed wavelength up to $200 \mathrm{~K}$ and one with a monotonic increase of wavelength with temperature. In their athermal temperature regime, the pattern wavelength is of the order of the impact diameter; it increases with ion energy and is flux independent.

However, van Dijken et al. report also intriguing differences compared to the present case: the wavelength of their 
patterns does not depend on ion fluence nor does the amplitude of their patterns exceed two layers for ion fluences up to $1.8 \times 10^{20}$ ions $/ \mathrm{m}^{2}$. For the grazing-incidence ion bombardment of $\mathrm{Pt}(111)$, however, a strong increase of the pattern wavelength with ion fluence (coarsening) and a power law increase of roughness with ion fluence is found. ${ }^{35}$

In their explanation of pattern formation, the authors point out the relevance of preferential erosion of steps illuminated by the ion beam as well as the importance of the depletion effect for ordering normal to the ion beam direction, in full agreement with the present findings. For the conditions used here, however, the athermal interaction of neighboring vacancy grooves by adatom repulsion and the positional alignment of vacancy islands due to subsurface channeling must be considered as additional important ingredients for pattern formation and ordering.

The transition from the athermal to the thermal pattern formation regime is attributed by van Dijken et al. to the onset of vacancy mobility. This is in striking contrast to the present study, which shows quite clearly the onset of full step edge mobility as the origin of the transition. The high temperature limit of pattern formation is attributed by van Dijken et al. to thermodynamics, striving for equilibrium shaped islands, while in the present case the initial formation of compact islands with close to equilibrium shapes does not prohibit pattern formation. Only the formation of an adatom lattice gas inhibits pattern formation by suppressing roughness buildup. Contrary to the present findings, van Dijken et al. claim that grooves develop even in the absence of thermally activated vacancy or adatom diffusion, and that thermally activated ledge atom diffusion is crucial for pattern formation.

The hierarchy of diffusion processes with their ladder of onset temperatures depends critically on the symmetry of the crystal surface [e.g., on fcc(001) surfaces step edge diffusion has typically a lower activation energy than adatom diffusion, which is just contrary to fcc(111) surfaces]. One possibility to account for the differences observed in the two systems is to attribute them to differences in the hierarchy of diffusion processes. The missing roughness increase with ion fluence on $\mathrm{Cu}(001)$, for instance, could possibly be related to a small or negligible step edge barrier for vacancies. Future research is certainly necessary to clarify the discrepancies between the two systems.

\section{ROUGHNESS EVOLUTION DURING GRAZING-INCIDENCE ION BOMBARDMENT}

Although a full discussion of the temperature and fluence dependence of the roughness evolution during grazingincidence ion bombardment is postponed to a future publication, two questions need to be answered in the present context. First, it has to be discussed how the initial vacancy groove pattern with a monolayer amplitude is transformed to subsequent layers and why the surface becomes rougher and rougher, yielding eventually a ripple pattern. Second, the bell shaped roughness curve of Fig. 4(a) demands an explanation.

In order to answer the question after the transfer of the vacancy groove pattern into a ripple pattern we consider a situation after the removal of one or several monolayers, where already a large step concentration is present [e.g., Figs. 6(a)-6(b) or Figs. 1(e)-1(f) of Ref. 36]. In such a situation new vacancy islands in lower layers are almost exclusively formed by steps traveling in the direction of the ion beam (we disregard for simplicity channeling effects). During its motion, occasionally massive step edge impacts will cause the formation of a large vacancy cluster in the level below the retracting step (compare Fig. 2). If this vacancy cluster survives and is enlarged by subsequent step edge impacts, erosion of a new layer starts. This survival probability is very different for a vacancy cluster formed in a vacancy groove (or on the bottom of a valley) and on an adatom band (or on the ridge of a ripple). In a vacancy groove the adatoms created by the massive impact will largely incorporate in the nearby ascending steps, while the vacancies remain at the bottom of the groove. Due to the step edge barrier for vacancies, their healing by ascending steps is hampered. It is thus likely that the vacancy cluster transforms by additional step edge impacts into a stable vacancy island located one layer below the retracting step that initiated its birth. On a retracting adatom band, the adatoms generated will behave similar as in a vacancy groove. They will largely incorporate into nearby descending steps (due to a negligible adatom step edge barrier). The vacancies, however, may heal without any additional barrier to the same descending steps as the adatoms. Thus, the chance for the survival of the cluster and its enlargement to a stable island is strongly diminished. A close inspection of the morphology in Fig. 6(b) reveals the dramatically different vacancy island formation rate within vacancy grooves and adatom bands. In conclusion, due to the step edge barrier for vacancies, the formation of new vacancy islands is much more likely in the ripple valleys than on the ripple ridges, thereby driving an increase of ripple amplitude with ion fluence.

The bell-shaped roughness curve of Fig. 4(a) exhibits at low temperatures very little roughness as the pattern formation is buried in noise. Defects are not separated by diffusion and with increasing ion fluence very quickly a saturation roughness develops. Locations of large roughness are even smoothed out by thermal spikes and the ensuing viscous flow. At high temperatures the roughness is low due to the lattice gas formation, which bypasses step edge barrier formation. At intermediate temperatures diffusion allows lateral separation of defects (depletion effect) and the step edge barrier to act. Thus the roughness increases continuously with ion fluence.

\section{CONCLUSION}

In conclusion, the analysis of ripple pattern formation under grazing-incidence ion bombardment indicates a distinction between an athermal $(200-500 \mathrm{~K})$ and a thermal $(500-700 \mathrm{~K})$ pattern formation regime. Below $200 \mathrm{~K}$ and above $700 \mathrm{~K}$, pattern formation ceases due to the dominance of noise and due adatom lattice gas formation, respectively. The transition between the thermal and the athermal pattern formation is triggered by the onset of full step edge mobility. Three elements determine the pattern formation in the ather- 
mal regime: the rapid motion of illuminated step edges in the ion beam direction, leading to vacancy grooves, the interaction of neighboring grooves through adatom repulsion, and the depletion effect preventing vacancy groove formation on narrow adatom bands separating vacancy grooves. While the first element causes the alignment of vacancy structures along the beam direction, the two latter give rise to ordering in the direction normal to the ion beam. In the thermal pattern formation regime alignment of vacancy structures is due to the preferential coalescence of compact vacancy clusters in the beam direction: This preferential coalecence may be traced back to positional alignment of compact vacancy islands in beam direction, caused by damage effects of planar subsurface channeling. The ordering in the direction normal to the ion beam is delayed compared to the athermal regime, as due to the larger wavelength adatom repulsion is of only small importance. In this regime ordering is supported by effective damage separation within vacancy grooves and the depletion effect.

The transfer of the initial patterns into well developed ripple patterns of larger roughness is due to the preferential production of new stable vacancy structures on the bottom of vacancy grooves. The origin of this preference is a large step edge barrier for vacancies to heal at ascending steps.

\section{ACKNOWLEDGMENTS}

This work was supported in part by the Deutsche Forschungsgemeinschaft via the project "Streifender Ionenbeschuss auf Metalloberflächen." The authors acknowledge useful discussions with Celia Polop and Joachim Krug. Y.R. is grateful for a grant from the TPSDP Ministry of National Education, Republic of Indonesia.
*Present address: Institut für Festkörperforschung, Forschungszentrum Jülich, 52425 Jülich, Germany.

'Present address: Solid State Physics, University of Twente, P.O.Box 217, 7500 AE Enschede, The Netherlands.

${ }^{1}$ Y. J. Chen, J. P. Wang, E. W. Soo, L. Wu, and T. C. Chong, J. Appl. Phys. 91, 7323 (2002).

${ }^{2}$ R. Moroni, D. Sekiba, F. Buatier de Mongeot, G. Gonella, C. Boragno, L. Mattera, and U. Valbusa, Phys. Rev. Lett. 91, 167207 (2003).

${ }^{3}$ P. Chaudhari, J. Lacey, J. Doyle, E. Galligan, S.-C. A. Lien et al., Nature 411, 56 (2001).

${ }^{4}$ L. Vattuone, U. Burghaus, L. Savio, M. Rocca, G. Costantini, F. Buatier de Mongeot, C. Boragno, S. Rusponi, and U. Valbusa, J. Chem. Phys. 115, 3346 (2001).

${ }^{5}$ S. Facsko, T. Dekorsy, C. Koerdt, C. Trappe, H. Kurz, A. Vogt, and H. L. Hartnagel, Science 285, 1551 (1999).

${ }^{6}$ K. C. Ruthe and S. A. Barnett, Surf. Sci. 538, L460 (2003).

${ }^{7}$ O. Azzaroni, P. L. Schilardi, R. C. Salvarezza, R. Gago, and L. Vázquez, Appl. Phys. Lett. 82, 457 (2003).

${ }^{8}$ U. Valbusa, C. Boragno, and F. Buatier de Mongeot, Mater. Sci. Eng., C 23, 201 (2003).

${ }^{9}$ A. Molle, F. B. de Mongeot, A. Molinari, F. Xiaerding, C. Boragno, and U. Valbusa, Phys. Rev. Lett. 93, 256103 (2004).

${ }^{10}$ O. Malis, J. D. Brock, R. L. Headrick, M.-S. Yi, and J. M. Pomeroy, Phys. Rev. B 66, 035408 (2002).

${ }^{11}$ P. Broekmann, A. Mewe, H. Wormeester, and B. Poelsema, Phys. Rev. Lett. 89, 146102 (2002).

${ }^{12}$ G. Costantini, F. B. de Mongeot, C. Boragno, and U. Valbusa, Phys. Rev. Lett. 86, 838 (2001).

${ }^{13}$ T. Michely, M. Kalff, G. Comsa, M. Strobel, and K. H. Heinig, Phys. Rev. Lett. 86, 2589 (2001).

${ }^{14}$ G. Costantini, S. Rusponi, R. Gianotti, C. Boragno, and U. Valbusa, Surf. Sci. 416, 245 (1998).

${ }^{15}$ M. V. Murty, T. Curcic, A. Judy, B. H. Cooper, A. R. Woll, J. D. Brock, S. Kycia, and R. L. Headrick, Phys. Rev. Lett. 80, 4713 (1998).

${ }^{16}$ T. Michely and G. Comsa, Nucl. Instrum. Methods Phys. Res. B 82, 207 (1993).
${ }^{17}$ T. Michely and G. Comsa, Surf. Sci. 256, 217 (1991).

${ }^{18}$ B. Poelsema, R. Kunkel, L. K. Verheij, and G. Comsa, Phys. Rev. B 41, R11609 (1990).

${ }^{19}$ D. Cherns, Philos. Mag. 36, 1429 (1977).

${ }^{20}$ S. J. Chey, J. E. Van Nostrand, and D. G. Cahill, Phys. Rev. B 52, 16696 (1995).

${ }^{21}$ S. Facsko, T. Bobek, H. Kurz, T. Dekorsy, S. Kyrsta, and R. Cremer, Appl. Phys. Lett. 80, 130 (2002).

${ }^{22}$ F. Frost and B. Rauschenbach, Appl. Phys. A: Mater. Sci. Process. 77, 1 (2003).

${ }^{23}$ F. Frost, A. Schindler, and F. Bigl, Phys. Rev. Lett. 85, 4116 (2000).

${ }^{24}$ S. Facsko, T. Bobek, A. Stahl, H. Kurz, and T. Dekorsy, Phys. Rev. B 69, 153412 (2004).

${ }^{25}$ S. Habenicht, Phys. Rev. B 63, 125419 (2001).

${ }^{26}$ W. L. Chan, N. Pavenayotin, and E. Chason, Phys. Rev. B 69, 245413 (2004).

${ }^{27}$ S. Rusponi, G. Costantini, C. Boragno, and U. Valbusa, Phys. Rev. Lett. 81, 2735 (1998).

${ }^{28}$ P. Karmakar and D. Ghose, Surf. Sci. 554, L101 (2004).

${ }^{29}$ R. M. Bradley and J. M. E. Harper, J. Vac. Sci. Technol. A 6, 2390 (1988).

${ }^{30}$ M. M. Makeev, R. Cuerno, and A. L. Barabasi, Nucl. Instrum. Methods 197, 185 (2002).

${ }^{31}$ T. Aste and U. Valbusa, Physica A 332, 548 (2004).

${ }^{32}$ R. Cuerno and A. L. Barabasi, Phys. Rev. Lett. 74, 4746 (1995).

${ }^{33}$ P. Sigmund, J. Mater. Sci. 8, 1545 (1973).

${ }^{34}$ S. van Dijken, D. de Bruin, and B. Poelsema, Phys. Rev. Lett. 86, 4608 (2001).

${ }^{35}$ H. Hansen, S. Meßlinger, G. Stoian, and T. Michely, in preparation.

${ }^{36}$ A. Redinger, H. Hansen, U. Linke, Y. Rosandi, H. M. Urbassek, and T. Michely, Phys. Rev. Lett. 96, 106103 (2006).

${ }^{37}$ M. Bott, T. Michely, and G. Comsa, Rev. Sci. Instrum. 66, 4135 (1995).

${ }^{38}$ A. Friedrich and H. M. Urbassek, Surf. Sci. 547, 315 (2003).

${ }^{39}$ H. Hansen, C. Polop, T. Michely, A. Friedrich, and H. M. Urbassek, Phys. Rev. Lett. 92, 246106 (2004). 
${ }^{40}$ C. Busse, C. Engin, H. Hansen, U. Linke, T. Michely, and H. M. Urbassek, Surf. Sci. 488, 346 (2001).

${ }^{41}$ H. Gades and H. M. Urbassek, Phys. Rev. B 50, 11167 (1994).

${ }^{42}$ J. F. Ziegler, J. P. Biersack, and U. Littmark, The Stopping and Range of Ions in Solids (Pergamon, New York, 1985).

${ }^{43}$ H. Winter, Phys. Rep. 5, 387 (2002).

${ }^{44}$ The change in onset temperature $\Delta T$ due to a different experimental time scale $\tau_{e}$ may be calculated according to $\Delta T$ $=T_{0} \cdot \ln n /(\ln m+\ln n)$ with the dimensionless factors $n=\tau_{e} / \tau_{0}$ and $m=\nu_{0} / 1 \mathrm{~s}^{-1}$. For lack of more knowledge, it is common to use the numerical value $5 \times 10^{12} \mathrm{~s}^{-1}$ for the attempt frequency $\nu_{0}$, which is of the order of a phonon frequency. As an example, for a time scale $\tau_{d}=20 \mathrm{~s}$, a decrease of $\Delta T=48 \mathrm{~K}$ results for $T_{0}$ compared to $\tau_{d}=1 \mathrm{~s}$ at $500 \mathrm{~K}$.

${ }^{45}$ W. Ehrhardt, in Landolt-Börnstein, New Series, Group III, Vol. 25, Atomic Defects in Metals (Springer-Verlag, Berlin, 1991), p. $88 \mathrm{ff}$.

${ }^{46}$ M. Morgenstern, T. Michely, and G. Comsa, Phys. Rev. Lett. 79, 1305 (1997).

${ }^{47}$ M. Morgenstern, T. Michely, and G. Comsa, Philos. Mag. A 79, 775 (1999).

${ }^{48}$ M. Bott, M. Hohage, M. Morgenstern, T. Michely, and G. Comsa, Phys. Rev. Lett. 76, 1304 (1996).

${ }^{49}$ K. Kyuno, A. Gölzhäuser, and G. Ehrlich, Surf. Sci. 397, 191 (1998).

${ }^{50}$ K. Kyuno and G. Ehrlich, Surf. Sci. 437, 29 (1999).

${ }^{51}$ B. Poelsema, K. Lenz, L. S. Brown, L. K. Verheij, and G. Comsa, Surf. Sci. 162, 1011 (1985).

${ }^{52}$ M. Hohage, M. Bott, M. Morgenstern, Z. Zhang, T. Michely, and G. Comsa, Phys. Rev. Lett. 76, 2366 (1996).

${ }^{53}$ G. L. Kellogg, J. Phys. (Paris) 47, C2-331 (1986).

${ }^{54}$ D. W. Bassett and P. R. Webber, Surf. Sci. 70, 520 (1978).

${ }^{55}$ M. Kalff, P. Šmilauer, G. Comsa, and T. Michely, Surf. Sci. Lett.
426, 447 (1999).

${ }^{56}$ M. Kalff, Jül-Bericht 3625, Forschungszentrum Jülich, Jülich 1999.

${ }^{57}$ M. Giesen, G. Schulze Icking-Konert, D. Stapel, and H. Ibach, Surf. Sci. 366, 229 (1996).

${ }^{58}$ T. Michely, T. Land, U. Littmark, and G. Comsa, Surf. Sci. 272, 217 (1992).

${ }^{59}$ M. Kalff, G. Comsa, and T. Michely, Surf. Sci. 486, 103 (2001).

${ }^{60} \mathrm{~T}$. Michely and J. Krug, Islands, Mounds and Atoms, Springer Series in Surf. Sci. Vol. 42 (Springer-Verlag, Berlin, 2004), p. $158 \mathrm{ff}$.

${ }^{61}$ K. Kyuno and G. Ehrlich, Phys. Rev. Lett. 81, 5592 (1998).

${ }^{62}$ U. Valbusa, C. Boragno, and F. B. de Mongeot, J. Phys.: Condens. Matter 14, 8153 (2002).

${ }^{63}$ Although the onset of full step edge mobility defines the onset of wavelength increase, it is likely (specifically at higher temperatures) that nucleation of the initial vacancy islands from the terrace damage becomes difficult (implying a low initial island density) and contributes to the wavelength increase. Consistent with the assumption that several diffusion processes with different effective activation energies are involved in the wavelength increase is the finding that a plot of $\ln \lambda$ vs $1 / T$ does not yield a straight line, as would be expected for a mechanism with a single activation energy.

${ }^{64}$ F. A. Nichols and W. W. Mullins, J. Appl. Phys. 36, 1826 (1965).

${ }^{65}$ F. A. Nichols, J. Appl. Phys. 37, 2805 (1966).

${ }^{66}$ M. Eßer, K. Morgenstern, G. Rosenfeld, and G. Comsa, Surf. Sci. 402, 341 (1998).

${ }^{67}$ B. Yang, J. Scheidtmann, J. Mayer, M. Wuttig, and T. Michely, Surf. Sci. 497, 100 (2002).

${ }^{68}$ E. Hahn, H. Schief, V. Marsico, A. Fricke, and K. Kern, Phys. Rev. Lett. 72, 3378 (1994).

${ }^{69}$ A. Friedrich, Diploma thesis, University Kaiserslautern, 2003. 